\title{
Les émeutes en Afrique du Sud : la stratégie de Buthelezi
}

\section{Philippe Chapleau}

\section{(2) OpenEdition \\ 1 Journals}

\section{Édition électronique}

URL : http://journals.openedition.org/conflits/137

DOI : $10.4000 /$ conflits. 137

ISSN : $1777-5345$

Éditeur :

CCLS - Centre d'études sur les conflits lilberté et sécurité, L'Harmattan

Édition imprimée

Date de publication : 21 mai 1992

ISSN : 1157-996X

Référence électronique

Philippe Chapleau, "Les émeutes en Afrique du Sud : la stratégie de Buthelezi », Cultures \& Conflits [En ligne], 05 | printemps 1992, mis en ligne le 13 mars 2006, consulté le 30 mars 2021. URL : http:// journals.openedition.org/conflits/137 ; DOI : https://doi.org/10.4000/conflits.137

Ce document a été généré automatiquement le 30 mars 2021.

Creative Commons License 


\title{
Les émeutes en Afrique du Sud : la stratégie de Buthelezi
}

\author{
Philippe Chapleau
}

1 Alors qu'on s'approche de la résolution de la crise constitutionnelle en Afrique du Sud, la violence reste endémique autour de Durban et de Johannesburg. Il est clair que ni la libération des chefs historiques de l'opposition noire ni la normalisation de l'activité politique n'ont permis de réduire la fréquence et l'ampleur de ce que la terminologie officielle qualifie de "troubles" (unrest). Quelque 3500 morts "politiques" en 1990, plus de 2600 en 1991 : les pertes en vies humaines sont plus élevées qu'au plus fort des émeutes de 1976-1977 ou qu'au cours de la période de crise de 1985 à 1988. Pourtant les activités terroristes ont cessé après l'acceptation par l'ANC d'une suspension de la lutte armée ; la répression a considérablement décru et les exécutions capitales ont cessé.

2 L'analyse des données numérique ${ }^{1}$ permet de mettre à jour un facteur nouveau dont l'émergence remonte à 1987 mais qui ne joue à plein que depuis 1990: les affrontements intracommunautaires en milieu noir.

3 L'ironie de cette situation n'a pas échappé aux observateurs dont Matthew Kentridge, auteur de An Unofficial War : "Dans la RSA de 1990, où rien n'est plus comme cela l'était quelques mois plus tôt, la seule source de tension permanente reste le conflit endémique entre Inkatha et l'UDF au Natal" ${ }^{2}$. Deux ans plus tard, la remarque de Kentridge vaut toujours mais mérite d'être réactualisée sur deux points: le conflit entre Inkatha et l'ANC a gagné le transvaal.

Ces affrontements intracommunautaires coûtent cher : en vies humaines (6 000 morts en 5 ans), en ressources gaspillées (l'équivalent de 400 millions de FF selon les assurances), en bâtiments à reconstruire ( 3 ou 4000 rien que pour le Natal). "Ce n'est ni l'Irlande du Nord ni le Vietnam, précisait John Aitchison de l'Université de Pietermaritzburg. ça ne ressemble pas non plus aux émeutes de la faim dans le tiersmonde et encore moins aux émeutes qui ont affecté les townships jusqu'en $1989 \Sigma$ Pendant 30 ans, le rejet du système a servi de moteur à la violence. Alors que ce système s'effondre et que l'ère de la répression se termine, on assiste à l'éclatement de nouvelles émeutes, d'émeutes "civiles" qui ne trouvent leur genèse ni dans la situation 
économique ni dans les antagonismes tribaux. Mais on fait souvent prévaloir ces deux types d'explication.

5 L'interprétation socio-économique

6 L'imposition d'états d'urgence au cours des années 60 , 70 et 80 n'a jamais été motivée par la menace terroriste et l'offensive lancée par MK (Umkontho we Sizwe, l'aile armée de l'ANC) contre les structures administratives, économiques ou militaires du régime nationaliste blanc. Ces états d'urgence ont été imposés en réponse à des vagues de contestation, de manifestations et d'émeutes qui n'avaient pour finalité que d'exprimer les revendications des communautés noires et métisses et de forcer les autorités à amender une Constitution bâtie sur le Population Reg-istration Act. Pas de mouvements concertés cependant à l'origine de ces actions de masse. Après 1960 et l'échec de la campagne nationale contre le Pass (qui réglementait les droits de séjour des Africains en zones blanches), les déclencheurs d'émeutes sont apparus plus localisés. Ainsi en juin 1976, ce sont les revendications des lycéens de Soweto qui ont déclenché l'une des plus graves crise de l'histoire du pays. Le 16 juin, une patrouille de la police, pour se dégager, a dû tirer sur quelques centaines d'adolescents qui manifestaient contre l'imposition de l'afrikaans comme langue d'enseignement. A cette manifestation succédaient des pillages, des émeutes sur l'ensemble des townships du Reef (la région de Johannesburg) puis des vagues de troubles sur l'ensemble du pays au cours des 6 mois suivants. A suivi une répression massive, le régime blanc en profitant pour décapiter les mouvements de la Conscience Noire et neutraliser l'activiste Steve Biko.

7 Scénario identique 8 ans plus tard au Cap. C'est cette fois la résistance des squatters du camps de Crossroads qui a servi de déclencheur. Insalubre, trop proche des zones blanches, le camp devait être détruit et ses habitants transférés. Le mouvement de protestation a vite débouché sur des affrontements avec la police puis avec les groupes conservateurs noirs - les Vigilantes - contrôlés par les autorités.

Dernier exemple, celui des ghettos noirs du Transvaal à partir de 1987. L'incapacité des administrations locales noires à gérer les townships et la corruption notoire des conseillers municipaux ont provoqué de graves émeutes et une vague d'assassinats (le supplice du Necklace, un pneu enflammé passé autour du cou a alors fait son apparition). En quelques semaines, la plupart des administrations locales cessaient de fonctionner (de 60 à $90 \%$ d'entre elles selon les zones et les démissions de conseillers municipaux se succédaient). "Une expulsion, une très longue attente a un robinet desservant 700 familles suffisent pour que le processus soit lancé, constatait un observateur du Joint Management System de Sandton en 1987. Cris, attroupement, jets de pierre, intervention maladroite de la police : l'émeute est déjà à son stade initiale et ne peut plus être enrayée que par une action massive des forces de sécurité. Mais la saturation des townships par la SADF ne peut être que ponctuelle. La solution réside dans une amélioration radicale des conditions de vie dans les townships". Au cours de cette période de crise socio-politique, l'ampleur de la répression et la récupération politique, par l'UDF ou la COSATU entre autres, suffisaient pour que la quasi-totalité du pays s'embrase et que le champ de revendications s'élargisse. Mais si à terme, on assistait à une condamnation du système, les mises à feu des émeutes apparaissaient clairement d'ordre social ou économique.

9 Crise du logement, effondrement des services municipaux, coupures d'eau ou d'électricité, inexistence des transports en commun, hausse des loyers, expulsions, crise de 1 'enseignement $\Sigma$ : les déclencheurs des émeutes des années 60, 70 et 80 
existent toujours et les deux foyers majeurs de conflictualité des années 90 - l'axe Durban/Pietermaritzburg et la région johannesbourgeoise - sont affectés par toutes ces carences.

D'autres carences se sont même développées. L'abandon de l'Influx Control par l'administration Botha a permis à des milliers de ruraux de gagner les zones urbaines autour desquelles se concentre l'essentiel de l'activité économique ${ }^{3}$. Ces arrivées massives ont exacerbé certains problèmes que la crise économique qui frappe la RSA depuis le milieu des années 80 avait fait émerger La dérivation d'une part importante du budget national à des fins militaires a retardé de nombreux programmes de développement dont devaient bénéficier les Noirs. Ainsi, l'aménagement des townships en eau courante et électricité est encore embryonnaire. La crise du logement a provoqué l'apparition de camps de squatters fortement politisés. Enfin, avec l'arrivée de migrants, le taux de chômage autour des métropoles industrielles a grimpé, tout aussi rapidement que la criminalité. La violence : multiforme, spontanée, politique ou criminelle, est donc devenue le principal facteur d'instabilité en RSA.

La "Prétoriastroïka", même si elle a fait sortir le pays de l'impasse politique, n'a en somme réglé aucun problème social ou économique. Les défavorisés souffrent toujours des mêmes maux et frustrations. Les échéances sont les mêmes qu'il y a 5 ans. Logiquement, des émeutes à motifs économiques ou sociaux devraient éclater épisodiquement sur l'ensemble du territoire et s'inscrire dans le schéma classique: revendication-récupération-répression. Or l'observation des types d'affrontement ne permet en rien de confirmer cette thèse économiste. Actuellement les victimes de violences traditionnelles de la période pré-quatre-vingt-dix ne représentent plus que $10 \% \mathrm{du}$ total. En outre, les émeutes sont localisées géographiquement et sont intracommunautaires puisqu'elles mettent en lignes essentiellement des organisations ou des communautés noires. Cette dernière dimension a permis d'étayer une thèse ethnique et de réduire les violences à des affrontements tribaux. 
d'Afrique à cohabiter. La présence blanche et son rôle stabilisateur s'en trouvent ainsi légitimés. Dans le contexte sud-africain il n'est pas étonnant que cette thèse soit ardemment soutenue par l'aile conservatrices. Mais si l'on prend la phase initiale de ce conflit - celle qui à partir de 1987 a affecté le Natal - il apparaît que les deux camps regroupaient exclusivement des Zoulous et que le rôle joué par les Xhosas ou les Indiens était minime, et cela en dépit des accusations du chef Buthelezi qui dénonçait des "éléments déstabilisateurs venus du Transkei", en un mot des Xhosas. Après le transfert conflictuel vers le Reef (le plateau au sud-ouest du Transvaal où est situé Johannesburg), d'autres communautés noires ont été affectées (Shangaans soutenant Inkatha, Sothos ou Ndebeles appuyant les mouvements antigouvernementaux). La violence n'a donc pas été circonscrite aux deux seules tribus xhosa et zoulou en dépit de l'impression - donnée à dessein parfois - de guerre tribale. devenu un parti politique qu'en juillet 1990. Mais il était auparavant bien plus qu'une organisation culturelle. Inkatha contrôle l'assemblée législative du Kwazulu (la KLA) ainsi que l'administration de ce Homeland. Il a joué un rôle prépondérant lors de l'établissement du projet constitutionnel Indaba. Quant à son chef, il a l'envergure, sinon l'ambition, d'un grand leader politique. Jusqu'en 1990, Buthelezi est souvent apparu comme l'un des interlocuteurs noirs les plus valables aux yeux de l'Occident, et surtout aux yeux de la communauté blanche séduite par l'approche libérale, capitaliste et fédéraliste de cet ancien avocat.

Bien qu'Inkatha clame avoir sa place au sein des organisations anti-apartheid, ses détracteurs ont mené de violentes campagnes et dénoncé "la politique collaboratrice du bouffon Buthelezi". La participation active de Buthelezi au système des Homelands il est Premier ministre du Kwazulu - constitue l'un des points du contentieux qui oppose Inkatha et le Congrès national africain (ANC). Pourtant Buthelezi a appartenu à l'ANC. Pourtant Oliver Tambo dans les années 70, a cru que Buthelezi pouvait incarner en RSA le mouvement banni'. Mais les ambitions personnelles de Buthelezi et son opposition à la lutte armée devait conduire à un divorce politique en 1979. Depuis lors, même s'il a multiplié les contradictions Buthelezi est resté intraitable : il a dénoncé le terrorisme, s'est opposé aux sanctions, a critiqué les options économiques marxistes du PAC et de l'ANC et a exalté les particularismes culturels. Concurremment, Buthelezi a su - à travers la KLA - tirer parti de certaines ressources de l'apartheid et en particulier de la législahon sur la sûreté de l'état. En fait, toute décision d'exception (état d'urgence, censure, interdiction $\Sigma$ ) prise par le pouvoir central de Prétoria a été entérinée par la KLA. La répression des "éléments subversifs" a permis aux services de 
sécurité du Kwazulu de traquer les opposants à Inkatha et de neutraliser les responsables de l'UDF.

Second acteur, le United Democratic Front a été créé en 1983 pour regrouper, alors que le fer de lance de l'opposition noire était en exil, plusieurs centaines d'organisations civiques, religieuses communautaires, culturelles et syndicales. Aux yeux des politiciens blancs, l'UDF n'était que le bras interne de l'ANC et dès sa formation, le front anti-apartheid a été soumis à de fortes pressions, avant d'être interdit le 24 février 1988. Au Natal, l'UDF a dû faire face aux man uvres d'intimidation puis d'annihilation d'Inkatha dont la stratégie vise l'élimination totale des groupes progressistes de la Province ${ }^{5}$.

Après la légalisation, en février 90 , des principaux mouvements d'opposition noire, l'UDF a vu son rôle décroître et ses membres rejoindre l'ANC. En août 91, l'UDF s'est donc dissout, fondant en fait ses structures dans celles de l'ANC et adjoignant à la vieille garde de l'ANC une génération de militants éprouvés et issus de la contestation des années 80 .

Lors de la bataille pour le Natal, l'UDF a porté une part des responsabilités en refusant l'adhésion d'Inkatha et en cherchant la confrontation avec l'administration Buthelezi (grèves, boycotts désordres étudiantins $\Sigma$ ). Appuyé par la COSATU, la puissante confédération des syndicats sud-africains, l'UDF a activement recruté dans les deux centres d'activités de la Province: Durban et Pietermaritzburg, où se concentre l'essentiel de l'industrie et de la population ouvrière. Aux défis verbaux ("Buthelezi est un lépreux politique", disait Patrick Lekota) a succédé une campagne d'implantation dans le fief d'Inkatha qui tentait vainement jusqu'en 87 de rameuter les Zoulous passés à l'UDF.

Le dernier acteur, c'est le régime blanc dont l'instrument - la police - est devenu une troisième force dans le conflit. Stratégiquement, le régime blanc a intérêt à exacerber les querelles entre mouvements noirs. Tactiquement, il y a collusion entre certains milieux des forces de sécurité et Inkatha qui n'a jamais été affecté par les états d'urgence successifs et les vagues de répression politique. Au Natal, Inkatha est ainsi devenu l'auxiliaire du système répressif en menant sa propre lutte contre l'opposition noire ${ }^{6}$.

La bataille pour le Natal

Malgré l'atroce réalité quotidienne des embuscades sur les bus, des luttes grenadées et des cadavres mutilés au fond des vallées et dans les rues des townships d'Edendale, la bataille pour le Natal peut se résumer en quelques dates.

- 1983-1985 : l'UDF, basé à Durban, poursuit son implantation au Natal et lance des mouvements de protestation visant l'administration provinciale, les autorités du Kwazulu.

29

- 1985 : début de la répression indirecte. Des Escadrons de la mort traquent les syndicalistes et les responsables de l'UDF

30

- Août 1986 : pour contrer l'avance de l'UDF, Inkatha lance une campagne massive de recrutement. Les affrontements commencent, 60 morts en septembre, 83 en octobre, 61 en novembre et 113 en décembre, au total près de 400 victimes. 
- 1988 : la flambée de violence autour de Pietermaritzburg pousse au départ de 60.000 résidents. Les affrontements se multiplient. "La violence est maintenant criminelle", déclare la police après le déploiement de plusieurs centaines d'hommes. L'année se clôt, sans solution de paix et, avec 850 nouvelles victimes.

32

- 1989: en dépit de nombreuses initiatives pour pacifier le Natal, la violence se maintient à un niveau égal et parfois supérieur à celui de 1988. Plusieurs commissions d'enquête mettent en cause Inkatha et dénoncent certaines man cuvres policières de soutien au mouvement du chef Buthelezi.

- 1990 : Peu après sa libération, Mandela lance un appel pour la paix à Durban. Malgré cela, les combats s'intensifient, en mars on relèvera 180 victimes. L'armée sud-africaine déploie des unités rapatriées de Namibie, dont le 32ème bataillon. En mai ont lieu les premiers entretiens ANC-gouvernement. Buthelezi est oublié. En août, le Reef s'embrase. Août, septembre et décembre verront des affrontements impitoyables autour de Johannesburg. Prétoria-Witwatersrand-Vereeninging) et leur positionnement dans les Hostels (résidence pour travailleurs) et certains quartiers ne constituent qu'une phase de la man $_{c}$ uvre de noyautage opérée par Inkatha qui avait réussi précédemment grâce à cette méthode à s'approprier les rênes des organes municipaux dans certains ghettos noirs et en particulier à Soweto.

Dans le grand township au sud-ouest de Johannesburg, 80 \% des conseillers municipaux sont membres d'Inkatha ou inféodés au parti zoulou. Cela ne signifie pas que $80 \%$ de la population soutienne Buthelezi, puisque, lors des dernières élections municipales, on n'a relevé que $15 \%$ de votants, zoulous pour la plupart ou membres du mouvement Sophasonkwe, dirigé par M. Tshabalala, proche de Buthelezi. En lançant un appel à l'abstention l'UDF/ANC a donc permis à Inkatha d'opérer une réelle mainmise sur la police municipale, le budget et l'administration de Soweto. Ainsi établi, Inkatha a pu mener des pressions sur les résidents, en inquiéter certains, en déplacer d'autres et est devenu le seul interlocuteur de l'administration provinciale blanche.

Au cours des premiers mois de 1990, Buthelezi a dû prendre en compte certains paramètres nouveaux. Au Natal, bien que vainqueur sur le terrain, Inkatha était de plus en plus critique ${ }^{7}$. Depuis la libération de Nelson Mandela (11 février 1990), le chef zoulou voyait son rôle amenuisé et sa participation à la politique nationale compromise. En avril, le comité directeur d'Inkatha a donc élaboré une stratégie visant à la reconnaissance du mouvement zoulou par l'ANC et devant permettre la remise au premier plan de Buthelezi. L'acceptation de cette stratégie de confrontation, stratégie élaborée par la haute hiérarchie de la Ligue de la jeunesse, a provoqué la démission du modéré Oscar Dhloma, secrétaire général d'Inkatha et l'un des plus proches collaborateurs de Buthelezi. De mai à juillet, de l'armement a été acheminé vers le Reef ${ }^{8}$ et la propagande anti-ANC s'est intensifiée dans les Hostels. Un groupe-action,

Cultures \& Conflits, 05 | printemps 1992 
certainement formé et entraîné par la SADF en Namibie puis intégré aux services de sécurité du Kwazulu et bénéficiant sans doute du soutien de certains milieux policiers a gagné le Reef pour y mener des attaques aveugles sur les gares et les arrêts de bus et pour lancer des raids ponctuels sur les zones résidentielles noires pro-ANC, toutes ces actions devant par contrecoup déboucher sur de massifs affrontements entre habitants des townships et occupants zoulous des Hostels. La guerre du Reef a commencé fin juillet 90, à Sebokeng, $100 \mathrm{~km}$ au sud de Johannesburg. Deux mois plus tard on dénombrait 800 victimes, résidents "neutres" ou militants de l'ANC pour la plupart. Si les responsables d'Inkatha rejetaient la responsabilité des violences sur le mouvement de Nelson Mandela, sur le terrain, le développement des affrontements donnait une tout autre impression. Des tireurs inconnus attaquaient préalablement certaines zones à forte densité des townships et provoquaient le regroupement des résidents qui adressaient des travailleurs zoulous ou harcelaient les Hostels. La contre-attaque zoulou suivait immanquablement. Des impi (les régiments de la tradition militaire zoulou) de 500 à 2000 hommes, armés d'armes blanches et identifiables à leurs bandeaux rouges, lançaient des opérations punitives. Le cycle des violences était ainsi amorcé.

Comme on l'a vu, le haut degré de violence dans les ghettos noirs et les frustrations exacerbées par les problèmes socio-économiques concourent à accroître la disponibilité "opérationnelle" des belligérants mais ne constituent plus les détonateurs des émeutes. Si dans les stades initiaux de la violence, les résidents ne s'identifient pas systématiquement comme membres ou sympathisants de l'ANC, le principe de polarisation joue rapidement à plein. Quelle que soit leur origine tribale, les victimes de l'agression zoulou en arrivent à se ranger sous la bannière de l'ANC et à s'identifier aux "Comrades". Dans le camp zoulou, la mobilisation est structurée : allégeance à Inkatha, admission dans un Hostel ou un quartier contrôlé par Inkatha, préparation idéologique, intégration dans un impi.

\section{Le calcul de Buthelezi}

42 L'émeute dans de telles conditions, n'est plus l'expression d'un refus du système et d'un rejet de la société raciste. Alors que l'apartheid s'effondre, l'émeute devient un levier de reconnaissance (recognition), le moyen de pression qu'un mouvement marginalisé a choisi pour se propulser à l'avant de la scène politique. Ainsi ce n'est pas une volonté nihiliste qui est derrière l'émeute mais la recherche d'une exposition maximale d'une violence spectaculaire à la fois politique et médiatique (surtout depuis la levée de la censure et l'abrogation des mesures de contrôle sur la presse). Les affrontements se sont poursuivis tout au long de 1991. Les forces de l'ordre ne parvenaient ni à prévenir les batailles rangées ni à empêcher les attaques aveugles. Les offres de médiations se multipliaient. Pour l'ANC qui avait renoncé à la lutte armée en août 90, il ne restait plus que le compromis, l'acceptation d'un dialogue direct avec Buthelezi et ainsi la reconnaissance explicite de son poids ${ }^{9}$. Bien que dix fois remise, bien que n'ayant rien réglé, la rencontre Mandela/Buthelezi de janvier 91 a consacré une victoire du chef zoulou. Du duo Mandela/de Klerk, on est passé à la Troïka Mandela/de Klerk/Buthelezi, un Buthelezi non plus Premier ministre du Kwazulu (puisqu'il est conscient de la faillite irrémédiable de la politique des Bantoustans) mais chef de parti avec un programme politique et économique. La poursuite des violences, en dépit des initiatives des deux camps pour tempérer les ardeurs, pourrait laisser croire que les masses échappent au contrôle des partis concernés. Il est bien plus probable qu'Inkatha - ou tout au moins 
certains courants au sein du mouvement - entend maintenir la violence à un niveau suffisamment élevé pour que sa présence dans certains groupes de paix ou commissions d'enquête soit plus que jamais nécessaire et acceptée et pour que son rôle d'acteur essentiel puisse être totalement reconnu aussi bien à l'intérieur qu'à l'extérieur du pays.

Conclusion

La man ${ }_{c} u v r e$ du chef Buthelezi semble donc avoir réussi et lui a permis de redresser une situation politique rendue précaire par le positionnement de l'ANC et de son chef sur l'échiquier sud-africain. Pour forcer la reconnaissance de son parti, Buthelezi a recouru à la violence mais sans chercher à atteindre directement ses adversaires principaux. Il s'est en quelque sorte approprié la violence ambiante; il a imposé une polarisation extrême en jouant à fond sur des stéréotypes négatifs ${ }^{10}$ et en initiant une mobilisation nationaliste zoulou pour s'opposer au panafricanisme de l'ANC. La violence a payé. Elle s'est répandue et multipliée, a permis d'arracher de nouvelles concessions, de consolider les assises nationales

d'Inkatha mais a surtout accru la stature du chef Buthelezi qui s'est ainsi extirpé des limbes dans lesquels il s'était trouvé relégué en 1990. Le duel traditionnel - Blancs contre Noirs - a vécu. Buthelezi a imposé une joute à trois. Saura-t-il se démarquer du pouvoir blanc et dépasser ses ambitions personnelles pour proposer une alternative originale? Saura-t-il surtout mettre fin à la violence? Le risque est grand que le recours à l'affrontement, l'émeute et l'assassinat ne soit devenu une pratique éprouvée et payante pour Inkatha et ses chefs.

\section{NOTES}

1. Voir Approches polémologiques, FEDN 1991.

2. Matthew Kentridge, An Unofficial War, Le Cap : David Philip Publishers, p. 2.

3. Le démantèlement des Homelands pourrait provoquer un exode encore plus massif vers les villes. Le risque d'émeutes de la faim et "du logement" poserait un problème majeur.

4. Cf. Matthew Kentridge, op. cit., p. 217.

5. Parmi les mouvements visés, on compte même ce CONTRALESA le Congrès des chefs traditionnels!

6. Indicateur de cette collusion, le nombre d'incarcérations politiques au Natal entre juillet 87 et juillet 89 . On dénombre cinquante fois plus de militants de l'UDF que de membres de l'Inkatha.

7. Le rôle des War lords d'Inkatha a été révélé lors d'enquêtes : collecteurs de fonds, chefs de milices, ils représentent Buthelezi au niveau local. Leur participation lors de violences organisées a provoqué plusieurs arrestations.

8. Principalement des AK47 qui proviennent du Mozambique et transitent par le Zululand ou le Swaziland. 
9. On notera que jusqu'en décembre $90, l^{\prime}$ ANC a évité de mettre en cause Inkatha nommément et rejetait sur le régime blanc l'entière responsabilité des violences. 10. Complot communiste, manipulation de l'ANC par le PC sud-africain, menaces sur la culture zoulou, ruine d'une Economie contrôlée $\Sigma$

INDEX

Index chronologique : 1990 - 2000

Mots-clés : émeutes, professionels de la politique, répression, violence Index géographique : Afrique australe, Afrique du Sud (R.S.A.)

Thèmes : ANC 